\title{
Critical Care Nursing in Hong Kong
}

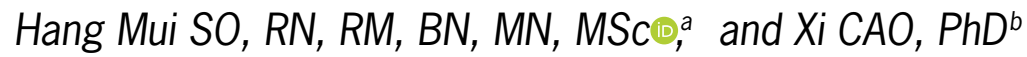

Background: Nursing shortage, especially in critical care unit, has become a big concern worldwide. Aim: This study aimed to provide the current states of critical care nursing in Hong Kong. Methods: A literature search was conducted. Findings were narratively summarized. Findings: Hong Kong faces an increasing demands in critical care service. However, the shortage of nursing manpower in ICU imposes challenges to meet such demands as well as the quality of critical care. Hospital authority has implemented various strategies such as the development of practice through Specialty Advisory Group (Critical Care) and Coordinating Committee in Intensive Care to address these issues. Conclusion: Efforts from all stakeholders are needed to meet the demands and improve the quality of critical care.

Keywords: critical care unit; nursing shortage; turnover; Hong Kong

\section{OVERVIEW OF INTENSIVE CARE UNITS IN HONG KONG}

The intensive care unit (ICU) refers to a specialized unit in a hospital to treat patients with severe and life-threatening illness requiring intensive monitoring, nursing care, and support of advanced technology equipment (Hong Kong Society of Critical Care Medicine, 2009). Because of the specialization of ICU, number of ICU bed is limited but its demand is increasing worldwide. The same condition is faced by Hong Kong which has a highly government subsidized healthcare system. In Hong Kong, there were 206 ICU beds in 2016 in public hospitals serving for 7.4 million residents (Yeung, 2016). Additionally, a high ICU occupancy rate $(>80 \%)$ is reported in nearly half of the major ICUs in Hong Kong, posing substantial strain on ICU capacity (Intensive Care National Audit \& Research Center [ICNARC], 2012). Despite this fact, the demand of ICU beds in Hong Kong is increasing due to its rapid ageing population, with an approximate 5\% increase from 2007 to 2012
(ICNARC, 2012). Additionally, the shortened length of hospital stay, an action to maximize ICU capacity and meet the demands of increasing number of critically ill patients, may result in early discharge of ill patients and thus increase the risk of ICU readmission (Wagner et al., 2013).

\section{ICU NURSING MANPOWER IN HONG KONG}

Concurrent with the increasing demands for ICU care services and advances in medical technologies, ICU requires more manpower to be operated in order to provide quality care. The Hospital Authority (HA), the major provider of public healthcare services in Hong Kong, roll out service enhancements for time-critical care to improve patients' outcomes, which then requires more critical care health professionals including nurses to fulfill this goal (Hospital Authority, 2017). However, Hong Kong is facing the same issue of ICU nurses shortage as other countries (Hospital Authority, 2017).

\footnotetext{
${ }^{a}$ Doctor of Nursing, Nurse Consultant (Intensive Care), Department of Intensive Care, Pamela Youde Nethersole Eastern Hospital, Hong Kong SAR, China

${ }^{b}$ Post-doctoral Fellow, The Nethersole School of Nursing, The Chinese University of Hong Kong, Hong Kong SAR, China.

E-mail:caoxi@cuhk.edu.hk
} 
Due to the intensive care services required by ICU patients, nurses in ICU usually have a heavy workload. It is estimated that the workload taking care of one ICU patient equals that of taking care of six to seven patients in a general ward (Yeung, 2016). Additionally, nurses working in critical care environments face increasing amount of work-related stress due to their exposure to human suffering, death, and family crises as well as the organizational constraints inherent in the hospital system (Hospital Authority, 2017). The heavy workload and the stressful experiences affect nurses' health and may increase their intention to leave ICU (Toh, Ang, \& Devi, 2012; Zhang et al., 2014). One ICU reported that $6.6 \%$ of ICU nurses left their job in $2015 / 16$, compared to the $3.9 \%$ in 2014/15 and 6.1\% in 2013/14 (Yeung, 2016). Such high turnover rate has greatly affected the ICU practice and the morale of nursing workforce in Hong Kong. Another issue faced by Hong Kong critical care nursing is experienced nurses seeking career promotions but limited senior nursing positions being offered in ICU compared with that in general wards, resulting in a continual drain of ICU-trained nurses (Hong Kong Society of Critical Care Medicine, 2009).

To fill the nursing manpower shortfall and improve quality of care, the Hong Kong Government and HA have launched a series of actions to retain and attract nurses, such as employing part-time nurses, recruiting allied healthcare assistants to assist registered nurses, improving work conditions, offering more flexible work hour arrangement, providing career progression opportunities such as offering additional promotion posts, and reemploy suitable retirees to address manpower needs (Hospital Authority, 2017). In addition to retain nurses, HA also added around 4,050 nurses with 280 senior nurses to the HA workforce during 2012 to 2017. As a result, the number of nurses has been increased by $19 \%$ from $2011 / 12$ (20, 901) to $2016 / 17$ (24, 959) (HKNC, 2017). Additionally, an attrition rate of $5.2 \%$ was reported in both 2011/12 and 2015/16 (Tsang, 2016), indicating the effective of the implemented strategies for nurses retention.
These strategies are applied for all clinical specialties including ICU. Additionally, to meet the increasing service demands for critical care service, the HA have taken various actions to expand ICU's capacity, such as by opening additional beds in the existing hospitals or through hospital redevelopment and expansion projects (Hospital Authority, 2017).

\section{QUALITY ENHANCEMENT OF CRITICAL CARE SERVICES FROM NURSES}

Despite the challenges critical care nurses are facing in Hong Kong, nurses are united to strive for excellence in practice and patient care. Specialty Advisory Group in Critical Care (SAG Critical Care) is established to fulfill this goal (Nursing Services Department, Hospital Authority Head Office, 2009). The SAG involves senior nursing managers and clinical expertise from all 15 ICUs in Hong Kong. These members include Departmental Operation Managers, Nurse Consultants, and Ward Managers. The SAG sets a platform to address issues related to ICU services, nursing workforce and training needs, quality assurance and profession standards, and information technology. It also serves a bridge to report and make recommendation to HA concerning the ICU services trend and development. Furthermore, nurse representatives from the SAG are members of the Coordinating Committee in Intensive Care (COC, ICU) too, which is a group of ICU expert doctors to discuss ICU services development plan in order to achieve more standardized service quality and treatment in all ICUs in Hong Kong to ensure patient safety. To enhance the morale of ICU nurses, the SAG has actively involved in various activities, such as negotiating the manpower issues with HA, supporting overseas and local training to uphold ICU nurses with related competency, advocating evidence-based nursing standards for provision of quality care to patients, piloting ICU outreach services to support continuity of post-ICU discharge care, and promoting transparency and knowledge sharing among all ICUs through organizing annual critical care forum. With these efforts, ICU nurses are more competent to provide quality patient care and 
meet the needs of critically ill patients at an international standard.

\section{References}

Chan, Z. C., Tam, W. S., Lung, M. K., Wong, W. Y., \& Chau, C. W. (2013). A systematic literature review of nurse shortage and the intention to leave. Journal of Nursing Management, 21(4), 605-613. doi:10.1111/j.13652834.2012.01437.x

Hong Kong Society of Critical Care Medicine. (2009). Development of ICU in Hong Kong. Retrieved from http://hksccm.org/index.php/ icus/39-development-of-icus-in-hong-kong/ 142-development-of-icu-in-hong-kong

Hospital Authority. (2017). Innovating for better care: Strategic plan 2017-2020. Retrieved from http://www.ha.org.hk/haho/ho/ap/HASP_1.pdf

Intensive Care National Audit \& Research Center. (2012). National and international benchmarked analysis of Hong Kong hospital authority intensive care unit data: Report on 2010 data (trends 2007-2010). Unpublished internal data

Legislative Council Panel on Health Service. (2018). Progress updates: Reviews on hospital authority and on healthcare manpower planning and professional development. Retrieved from https://www. legco.gov.hk/yr17-18/english/panels/hs/.../ hs20180115cb2-656-3-e.pdf

The Nursing Council of Hong Kong (HKNC). (2017). Statistics and lists of nurses. Retrieved from http://www.nchk.org.hk/ en/statistics_and_lists_of_nurses/statistics/ index.html

Nursing Services Department, Hospital Authority Head Office. (2009). Specialty advisory group. Retrieved from http://www. ha.org.hk/nurse/nursing/nursing.html
Toh, S. G., Ang, E., \& Devi, M. K. (2012). Systematic review on the relationship between the nursing shortage and job satisfaction, stress and burnout levels among nurses in oncology/haematology settings. International Journal of Evidence-based Healthcare, 1O(2), 126-141. doi:10.1111/ j.1744-1609.2012.00271.x

Tsang, E. (2016). Inside Hong Kong's public hospital crisis: Temporary beds angry patients, nurses and doctors stretched to breaking point. Retrieved from http://www.scmp.com/ news/hong-kong/health-environment/article/ 1931049/inside-hong-kongs-public-hospitalcrisis-temporary

Wagner, J., Gabler, N. B., Ratcliffe, S. J., Brown, S. E., Strom, B. L., \& Halpern, S. D. (2013). Outcomes among patients discharged from busy intensive care units. Annals of Internal Medicine, 159(7), 447-455. doi:10. 7326/0003-4819-159-7-201310010-00004

Yeung, C. (2016). Hospitals hit as top nurses quit for better jobs. The Standard. Retrieved from http://www.thestandard.com.hk/sectionnews.php?id=173136

Zhang, L. F., You, L. M., Liu, K., Zheng, J., Fang, J. B., Lu, M. M., \& Wu, X. (2014). The association of Chinese hospital work environment with nurse burnout, job satisfaction, and intention to leave. Nursing Outlook, 62(2), 128-137. doi:10.1016/j.outlook.2013.10.010

Disclosure. This study did not receive any finding. HMS contributed to the study design, literature search, and the writing of the first draft of the manuscript. XC contributed to the study design, literature search, and critical revision of the manuscript. All authors contributed to and have approved the final manuscript. 